\title{
Força muscular em idosos I - Será o treino generalizado suficientemente intenso para promover o aumento da força muscular em idosos de ambos os sexos?
}

\author{
J. Carvalho \\ J. Oliveira \\ J. Magalhães \\ A. Ascensão \\ J. Mota
}

J.M.C. Soares

https://doi.org/10.5628/rpcd.04.01.51

\section{RESUMO}

O principal objectivo deste trabalho foi determinar o efeito de um programa generalizado de actividade física, com a duração de 6 meses, na força máxima isocinética dos flexores e extensores do joelho em idosos homens e mulheres. Vinte e cinco idosos, 7 homens (idade $=68.1 \pm 2.8$ anos; altura $=1.60 \pm 0.81 \mathrm{~m}$; peso $=72.5 \pm 16.42 \mathrm{~kg}$ ) e 18 mulheres (idade média $=$

$69.5 \pm 4.2$ anos; altura média $=1.56 \pm 0.9 \mathrm{~m}$; peso médio $=69.8$ $\pm 10.6 \mathrm{~kg}$ ) foram submetidos durante 6 meses a um pro-grama bi-semanal de actividade física generalizada ("Ginástica de Manutenção" - 50 min.). A força máxima isocinética dos extensores e flexores do joelho foi avaliada, em todos os sujeitos, através de um dinamómetro isocinético (Biodex System 2, USA) em duas velocidades distintas $60^{\circ} / \mathrm{seg}$. (1.05 rad.sec. ${ }^{-1}$ ) e $180^{\circ}$ seg. (3.14 rad.sec ${ }^{-1}$ ), antes e depois do programa de treino. Os resultados mostraram não existirem alterações significativas ( $p>0.05$ ) após treino. Para além disso, apesar dos níveis de força dos homens terem sido sempre significativamente mais elevados comparativamente às mulheres, a percentagem de alteração após treino foi independente do género. Ou seja, nem as mulheres nem os homens apresentaram melhorias significativas na força muscular após 6 meses de treino generalizado. Neste sentido, os resultados deste trabalho sugerem que, 6 meses de treino generalizado, 2 vezes por semana, 50 minutos por dia parecem não se constituir como um estímulo suficientemente intenso para induzir alterações significativas na força muscular de idosos saudáveis.

Palavras-chave: envelhecimento, actividade física, força muscular, isocinético.
Faculdade de Ciências de Desporto e de Educação Física Universidade do Porto, Portugal

\author{
ABSTRACT \\ Aging and muscle strength I - Is generalized physical activity \\ adequate to improve muscular strength in elderly?
}

The main purpose of this study was to determine the effect of a 6month general physical activity program on knee extensor and flexor isokinetic peak torque of elderly adults according to gender. Twenty-five elderly volunteers, 7 men (age $=68.1 \pm 2.8$ years; height $=$

$1.60 \pm 0.81 \mathrm{~m}$; weight $=72.5 \pm 16.42 \mathrm{~kg})$ and 18 women $($ age $=$ $69.5 \pm 4.2$ years; height $=1.56 \pm 0.9 \mathrm{~m}$; weight $=69.8 \pm 10.6 \mathrm{~kg}$ ) were submitted to a general physical activity program ("Gymnastics") twice per week during 6 months. All the subjects were tested for maximal strength of quadriceps and hamstrings on an isokinetic dynamometer (Biodex system 2, USA) at $60^{\circ} / \mathrm{sec}$. (1.05 rad.sec. $\left.{ }^{-1}\right)$ and $180^{\circ} / \mathrm{sec}\left(3.14 \mathrm{rad} . \mathrm{sec}^{-1}\right)$ before and after the training period. The results show no significant changes $(p>0.05)$ after training. Moreover, although men are stronger than women in absolute strength, there is no apparent difference between men and women in their response to training. Neither men, nor women had presented significant improvements in muscle strength after 6 months of a generalized physical activity program. In this way, the findings of the present study showed that six months of generalised training, twice a week, 50 minutes per day, seem therefore not sufficient to induced marked changes in muscle strength of healthy and fit elderly people.

Key Words: aging, physical activity, muscle strength, isokinetic. 


\section{INTRODUÇÃO}

A capacidade de realizar diferentes actividades diárias, actividades laborais ou recreacionais é determinada, em grande parte, pela capacidade de desenvolver força muscular $(3,10)$. Diferentes autores têm referido que a atrofia e fraqueza musculares associadas ao envelhecimento são aspectos determinantes na morbilidade e mortalidade destes escalões etários mais velhos $(3,4,20)$. A perda da força e da massa muscular predispõe os idosos a uma limitação funcional, sendo este um factor predisponente para muitos dos processos patológicos associados ao aumento da morbilidade e mortalidade (3). Assim, das várias alterações fisiológicas induzidas pelo envelhecimento e/ou inactividade física, as alterações sobre o sistema muscular esquelético revelam-se de especial importância. Para além da sua relação com a mobilidade, funcionalidade e autonomia, a força tem igualmente um papel preponderante na diminuição do risco de quedas e, consequentemente, de fracturas facilitadas pela maior desmineralização óssea típica do idoso (4).

Numerosos estudos têm demonstrado que, com estímulos adequados de força, idosos de ambos os sexos apresentam ganhos na força muscular semelhantes, ou até relativamente superiores, àqueles encontrados nos jovens $(7,8,21,25)$. Todavia, a maioria destes estudos sobre o efeito da actividade física na força muscular dos idosos baseiam-se em protocolos de treino intensivo específico de funções isoladas, sendo menos frequentes os estudos que utilizam programas de actividade física generalizada, apesar de ser aquela mais vulgarmente aplicada a este escalão etário $(15,18)$.

Neste sentido, o objectivo principal deste trabalho foi o de avaliar o efeito de um programa generalizado de actividade física (i.e., "Ginástica de Manutenção") sobre a força muscular dos membros inferiores do idoso em função da variável sexo.

\section{MATERIAL E MÉTODOS}

\section{Amostra}

Foram inicialmente estudados 32 idosos voluntários, de idades compreendidas entre os 65 e os 80 anos. Todos os sujeitos eram sedentários, não tendo participado em qualquer tipo de actividade física formal, pelo menos, nos 5 anos que antecederam o estudo.
Foram excluídos os sujeitos que por razões de saúde ou de assiduidade não cumpriam os requisitos mínimos para o cumprimento do protocolo de avaliação, tendo sido considerados os seguintes critérios de exclusão: ausência de mais de $20 \%$ do total das sessões de actividade física $(n=3)$, a não-presença a mais de 8 sessões consecutivas $(n=3)$ e a existência de patologias significativas de foro ortopédico que obrigavam à utilização de qualquer meio auxiliar na locomoção $(n=1)$.

Assim, a amostra final passou a ser constituída por 25 indivíduos, com uma média de idade de $69.4 \pm$ 4.1 anos, um peso médio correspondente a $69.9 \pm$ $\pm 14.4 \mathrm{Kg}$ e uma altura média de $1.57 \pm 0.8 \mathrm{~m}$., dos quais 18 do sexo feminino e 7 do sexo masculino. As principais características de ambos os grupos (homens e mulheres) estão descritas no Quadro 1.

Quadro 1 - Principais características da amostra [média \pm desvio padrão].

\begin{tabular}{l|c|c|c} 
& Homens & Mulheres & $\mathrm{p}$ \\
\hline Idade (anos) & $68.1 \pm 2.8$ & $69.5 \pm 4.2$ & $\mathrm{~ns}$ \\
\hline Peso $(\mathrm{Kg})$ & $72.5 \pm 16.4$ & $68.8 \pm 10.6$ & $\mathrm{~ns}$ \\
\hline Altura $(\mathrm{m})$ & $1.60 \pm 0.8$ & $1.56 \pm 0.9$ & $\mathrm{~ns}$ \\
\hline $\mathrm{n}$ & 7 & 18 &
\end{tabular}

Todos os sujeitos da amostra eram voluntários tendo sido informados sobre os objectivos, procedimentos e possíveis implicações do protocolo experimental, após o que deram o seu consentimento verbal para participarem no estudo.

Os sujeitos que constituíram a amostra viviam de forma independente, realizando autonomamente as tarefas básicas diárias.

A presença de patologias crónicas e o uso de medicamentos foram determinados a partir de informação pessoal, assim como, por parte do respectivo médico assistente. À excepção de um sujeito nenhum dos restantes participantes apresentou quaisquer problemas de saúde que pudessem comprometer o protocolo experimental. De igual modo, depois de todos os fármacos terem sido analisados medicamente, foram considerados como não tendo influência nos parâmetros avaliados. 
Todos os elementos da amostra eram destros e não fumadores.

Foi pedido a todos os sujeitos amostra para manterem as suas actividades de rotina do dia a dia e para não alterarem o seu nível de actividade física ao longo do programa de treino. Com o objectivo de verificar as possíveis modificações dessa actividade diária, foi aplicado nos dois momentos de avaliação um inquérito baseado no questionário de Baecke et al. (1) e validado para a população idosa por Voorrips et al. (24).

\section{Protocolo de treino}

Todos os sujeitos da amostra foram submetidos a um programa bi-semanal de actividade física geral do tipo "Ginástica de Manutenção" durante 6 meses. As sessões foram supervisionadas por um monitor académica e profissionalmente qualificado, tendo a duração de 50 minutos.

As sessões foram, de um modo geral, constituídas por: (i) um período de aquecimento de aproximadamente 10 minutos onde se incluíram o caminhar, exercícios calisténicos e exercícios de flexibilidade; (ii) um trabalho muscular localizado de cerca de 15 minutos, onde no trabalho de força muscular sobre os membros inferiores se utilizou apenas o peso do corpo, ocorrendo, por vezes, estes exercícios em simultâneo com exercícios de força para os membros superiores; estes exercícios foram feitos recorrendo ao uso de diferentes tipos de material, tais como, pesos livres entre 1 e $3 \mathrm{Kg}$, bandas elásticas e com o recurso ao próprio peso corporal; o trabalho muscular tentou ser o mais individualizado possível sendo, na generalidade dos casos, realizadas 2 a 3 séries de 8 a 15 repetições dos principais grupos musculares, nomeadamente, da musculatura dos membros inferiores e superiores e músculos posturais; (iii) um trabalho aeróbio através de uma variedade de exercícios, tal como, "jogging”, caminhar e dançar; (vi) alguns exercícios de coordenação, jogos lúdicos e de equilíbrio; e, no final, um período de

relaxamento/alongamento englobando, fundamentalmente, exercícios respiratórios e de flexibilidade.

\section{Avaliação força isocinética}

A avaliação isocinética da força dos músculos extensores e flexores do joelho foi realizada em ambos os membros inferiores mediante a utilização de um dinamómetro (Biodex System 2, USA) antes (M0) e depois (M1) do programa de treino em duas velocidades distintas: 60\% $/ \mathrm{seg}$. (1.05 rad.seg. ${ }^{-1}$ ) e $180^{\circ} / \mathrm{seg}$ (3.14 rad.seg. $\left.{ }^{-1}\right)$.

O posicionamento do indivíduo e o alinhamento das articulações para a flexão/extensão do joelho foram efectuados de acordo com as instruções definidas para este equipamento pela Biodex Medical System, Inc (26). Após os indivíduos estarem confortavelmente sentados, procedeu-se à colocação das bandas bem ajustadas ao nível do tronco, bacia e coxa de modo a estabilizar estes segmentos corporais e restringir o mais possível o movimento à flexão e extensão do joelho.

O eixo de rotação do dinamómetro foi alinhado com o epicôndilo femoral e a carga de resistência foi colocada cerca de $2 \mathrm{~cm}$ acima do maléolo interno. A referência anatómica angular da articulação do joelho introduzida no dinamómetro foi obtida mediante a utilização de um goniómetro. Os possíveis erros induzidos no torque pela força da gravidade foram corrigidos com base no peso do membro inferior a $0 \% \mathrm{seg}$. e calculados pelo próprio "software" do equipamento. Antes da realização do teste máximo, os sujeitos realizaram um aquecimento estandardizado numa bicicleta ergométrica (Monark, Sweden) durante 5 minutos, a $60 \mathrm{rpm}$, utilizando, para tal, uma carga correspondente a $2 \%$ do peso corporal. Os sujeitos tiveram, ainda, um prévio período de habituação ao dinamómetro mediante a realização de dez repetições submáximas de extensão/flexão do joelho a $180^{\circ}$ seg. e cinco repetições a $60^{\circ} / \mathrm{seg}$., após o qual se seguiu um período de repouso de 2 minutos. Para o teste, os indivíduos efectuaram cinco repetições máximas a $180 \%$ seg. e três a $60^{\circ}$ /seg., havendo um período de repouso de 2 minutos entre os testes. Os sujeitos foram instruídos para exercerem o máximo de força possível, tanto na extensão, como na flexão do joelho.

Para a avaliação da força máxima, a totalidade do movimento do membro inferior foi requerida desde a posição de flectido $\left(90^{\circ}\right)$ até à máxima extensão possível. Durante o teste, os sujeitos foram verbalmente encorajados para desenvolverem a sua máxima força, não tendo, no entanto, quaisquer "feedbacks" visuais. 


\section{Procedimentos estatísticos}

Procedeu-se a uma análise exploratória dos dados com o objectivo de averiguar a normalidade da distribuição correspondente a cada uma das variáveis em estudo, assim como a presença de "outliers". A análise das diferenças entre os grupos e entre os dois momentos de avaliação foi efectuada a partir de, respectivamente, teste de Mann-Whitney e $t$-teste de medidas dependentes. O nível de significância considerado foi de $\mathrm{p}<0.05$.

Para determinação da fidelidade do teste, foi efectuada, em 8 sujeitos, a aplicação sucessiva do instrumento (teste e reteste) com espaçamento de 20 dias. O coeficiente de correlação obtido foi de $r=0.93$ $(\mathrm{p}<0.001)$.

\section{RESULTADOS}

Da análise dos Quadros 2 e 3 , podemos constatar que, à excepção da flexão do joelho do membro dominante a $180^{\circ} / \mathrm{seg}$. no momento inicial, as mulheres apresentam, em todas as variáveis, valores significativamente inferiores aos dos homens. No entanto, excluindo o movimento de flexão do membro dominante a $180^{\circ}$ /seg., não foram observadas diferenças com significado estatístico na percentagem de alteração após treino entre os dois sexos. De igual modo, é possível verificar que à excepção da flexão a $180^{\circ}$ seg. do membro dominante nos homens, o treino não induziu alterações significativas.

Quadro 2 - Peak Torque (PT, Nm] dos idosos homens $(n=7)$ e mulheres ( $n=18$ ] nos momentos inicial e final do treino e respectiva percentagem de alteração na velocidade angular de $180 \%$ seg. (média \pm desvio padrão).

\begin{tabular}{|c|c|c|c|c|c|}
\hline & & ED & END & FD & FND \\
\hline \multirow{4}{*}{$\begin{array}{l}\text { MO } \\
(\mathrm{Nm})\end{array}$} & \multirow{2}{*}{ Homens } & 90.5 & 74.9 & 38.8 & 47.5 \\
\hline & & \pm 10.5 & \pm 16.4 & \pm 17.1 & \pm 13.6 \\
\hline & \multirow{2}{*}{ Mulheres } & $56.6^{*}$ & $53.5^{*}$ & 29.3 & $30.4^{*}$ \\
\hline & & \pm 13.7 & \pm 7.9 & \pm 7.4 & \pm 6.7 \\
\hline \multirow{4}{*}{$\begin{array}{l}\text { M1 } \\
{[\mathrm{Nm}]}\end{array}$} & \multirow{2}{*}{ Homens } & 87.5 & 79.7 & 46.7\# & 45.1 \\
\hline & & \pm 15.2 & \pm 13.6 & \pm 15.9 & \pm 9.3 \\
\hline & \multirow{2}{*}{ Mulheres } & $56.2^{*}$ & $52.7^{*}$ & $33.3^{*}$ & $28.7^{*}$ \\
\hline & & \pm 10.8 & \pm 8.3 & \pm 7.3 & \pm 6.0 \\
\hline \multirow{4}{*}{$\begin{array}{l}\text { \% Altera- } \\
\text { ção }\end{array}$} & \multirow{2}{*}{ Homens } & -7.1 & 0.8 & $28.1^{*}$ & -1.7 \\
\hline & & \pm 10.8 & \pm 15.8 & \pm 13.4 & \pm 20.2 \\
\hline & \multirow{2}{*}{ Mulheres } & 1.8 & 0.1 & 6.9 & -8.3 \\
\hline & & \pm 20.2 & \pm 15.1 & \pm 22.3 & \pm 12.1 \\
\hline
\end{tabular}

$\varepsilon=$ extensão do joelho; $F=$ flexão do joelho; $D=$ membro dominante; $N D=$ membro não-dominante; * Homens vs. Mulheres, \# MO vs. M1 $(p<0.05)$
Quadro 3 - Peak Torque $(P T, N m$ ) dos idosos homens $(n=7)$ e mulheres $[n=$ 18) nos momentos inicial e final do treino e respectiva percentagem de alteração na velocidade angular de 60\% $\mathrm{seg}$. (média \pm desvio padrão).

\begin{tabular}{|c|c|c|c|c|c|}
\hline & & ED & END & FD & FND \\
\hline \multirow{4}{*}{$\begin{array}{l}\text { MO } \\
{[\mathrm{Nm}]}\end{array}$} & \multirow{2}{*}{ Homens } & 130.4 & 125.9 & 62.5 & 62.7 \\
\hline & & \pm 28.9 & \pm 37.9 & \pm 20.6 & \pm 16.4 \\
\hline & \multirow{2}{*}{ Mulheres } & $90.4^{*}$ & $85.8^{*}$ & $39.5^{*}$ & $39.4^{*}$ \\
\hline & & \pm 20.7 & \pm 15.1 & \pm 9.4 & \pm 7.8 \\
\hline \multirow{4}{*}{$\begin{array}{l}\mathrm{M} 1 \\
(\mathrm{Nm})\end{array}$} & \multirow{2}{*}{ Homens } & 126.2 & 123.6 & 61.5 & 59.5 \\
\hline & & \pm 32.2 & \pm 29.7 & \pm 20.6 & \pm 8.6 \\
\hline & \multirow{2}{*}{ Mulheres } & $88.1^{*}$ & $83.5^{*}$ & $41.3^{*}$ & $37.9 *$ \\
\hline & & \pm 15.1 & \pm 15.6 & \pm 11.7 & \pm 10.3 \\
\hline \multirow{4}{*}{$\begin{array}{l}\text { \% Altera- } \\
\text { ção }\end{array}$} & \multirow{2}{*}{ Homens } & -10.9 & -7.1 & -0.7 & -4.1 \\
\hline & & \pm 17.6 & \pm 17.1 & \pm 5.0 & \pm 15.7 \\
\hline & \multirow{2}{*}{ Mulheres } & 0.6 & -1.1 & 4.6 & -5.1 \\
\hline & & \pm 19.7 & \pm 13.1 & \pm 29.2 & \pm 18.8 \\
\hline
\end{tabular}

$\varepsilon=$ extensão do joelho; $F=$ flexão do joelho; $D=$ membro dominante; $N D=$ membro não-dominante; ${ }^{*}$ Homens vs. Mulheres, \# MO vs. M1 ( $\left.p<0.05\right)$.

\section{DISCUSSÃO}

Os resultados deste estudo mostram que, apesar das diferenças entre homens e mulheres nos níveis de força, a percentagem de alteração da força muscular isocinética com o treino não foi significativamente diferente entre os dois sexos, não apresentando, de um modo geral, nem as mulheres, nem os homens, alterações significativas com o programa de treino generalizado.

Apesar da abundância da informação quanto aos efeitos do treino no organismo humano, existe uma falha na estandardização na metodologia relativa ao protocolo de treino o que, por vezes, torna difícil a interpretação dos resultados (17).

Os ganhos de força têm sido observados com diferentes tipos de treino: isométrico, isocinético e isotónico $(7,8,21,25)$. Todavia, a maioria destes estudos sobre o efeito da actividade física na força muscular dos idosos, refere-se a treino específico de força com pesos ou equipamento específico, sendo menos frequentes os estudos que utilizam programas de actividade física generalizada, apesar de ser aquela mais vulgarmente aplicada a este escalão etário $(15,18)$

No nosso estudo, aplicámos um programa de actividade física generalizado cujo objectivo central foi a 
melhoria de todas as componentes da aptidão física com reflexo sobre a qualidade de vida dos idosos. A grande vantagem deste tipo de programas generalizados é o facto de serem mais motivadores do que os específicos de força e de, geralmente, reflectirem melhor as actividades diárias do idoso. De facto, a maioria das tarefas quotidianas, tais como caminhar, subir degraus, pegar em compras, etc., são actividades dinâmicas que requerem a coordenação e a contracção de vários grupos musculares simultaneamente. Neste sentido, para a melhoria da funcionalidade, é importante trabalhar as diferentes componentes da aptidão física através de movimentos multiarticulares e não apenas realizar exercícios de força muscular com movimentos articulares isolados.

De acordo com os resultados do torque máximo do presente estudo, podemos verificar que os valores não se alteraram significativamente após aplicação do programa bi-semanal de "Ginástica de Manutenção" sugerindo que, provavelmente, este tipo de actividade física generalizada não foi suficientemente intensa ou específica para provocar alterações significativas neste parâmetro independentemente do sexo.

Embora existam estudos que refiram a melhoria dos níveis de força com a actividade física generalizada $(14,18)$, a maioria dos trabalhos, tal como o presente estudo, referem a necessidade da especificidade do treino associada à elevada intensidade, para poderem ser observados aumentos na força e na massa musculares (5). Os treinos generalizados e com intensidades mais baixas (peso corporal, bandas elásticas, etc.) resultam, normalmente, em reduzidos ou nulos aumentos da força $(11,25)$.

Puggaard et al. (18) encontraram melhorias na força isométrica máxima de diferentes grupos musculares entre 7 e $27 \%$ após aplicação de um programa bi-semanal de treino generalizado (45 minutos) durante 5 meses. De acordo com os autores, este resultado está, provavelmente, relacionado com o baixo nível inicial dos seus idosos. Reforçando esta ideia, os autores evidenciam os maiores ganhos encontrados nos músculos do tronco, que são, geralmente, menos solicitados no dia a dia dos idosos, comparativamente aos músculos dos membros inferiores e superiores. Neste sentido, é também possível que o nível inicial dos sujeitos da amostra dos estudos que se referem à melhoria da força após actividade física generalizada (18) seja mais baixo do que o dos idosos do presente trabalho, possuindo, provavelmente, os idosos deste estudo níveis de força acima da média considerada para esta idade e, como tal, responderem de forma menos evidente a um dado estímulo de treino. De facto, no presente estudo, quer os homens, quer as mulheres apresentam valores iniciais de força superiores aos observados noutros estudos. Por exemplo, apenas os valores do torque da flexão do joelho nas mulheres apresentados por Salem et al. (19) na velocidade de $60 \%$ seg. no membro dominante são valores semelhantes aos deste trabalho $(39.8 \pm 14.6$ $\mathrm{Nm}$ vs. $39.5 \pm 9.4 \mathrm{Nm}$ ). Quer os valores no movimento de flexão nos homens $(5.6 \pm 20.9 \mathrm{Nm}$ vs. $62.5 \pm$ $20.6 \mathrm{Nm}$ ), quer os de extensão em ambos os sexos $(56.1 \pm 21.9 \mathrm{Nm}$ vs. $90.4 \pm 20.7 \mathrm{Nm}$ nas mulheres e $90.0 \pm 38.5 \mathrm{Nm}$ vs. $130.4 \pm 28.9 \mathrm{Nm}$ nos homens) descritos por estes autores são mais baixos comparativamente aos apresentados no presente trabalho. Reforçando a ideia do princípio da sobrecarga, onde os baixos níveis apresentam uma maior magnitude nos ganhos de força, verificamos que apenas foram encontrados aumentos significativos da força após treino no movimento que apresentou, no sexo masculino, valores inicialmente mais baixos.

Tal como observado no presente estudo, a literatura tem descrito que os homens são significativamente mais fortes, independentemente do grupo muscular avaliado e da velocidade angular envolvida $(6,10$, $13,22,23)$. No presente trabalho, o torque máximo do membro dominante no momento inicial das mulheres foi significativamente inferior ao dos homens, apresentando as mulheres, em média, valores na velocidade angular de $60 \%$ seg. de, respectivamente, $69 \%$ e $63.2 \%$ da força dos homens para os músculos extensores e flexores do joelho e de, respectivamente, $62.5 \%$ e $75 \%$ da força dos homens quando testadas a $180^{\circ} / \mathrm{seg}$. No estudo de Frontera et al. (6), as mulheres avaliadas a $60^{\circ} / \mathrm{seg}$. apresentaram, respectivamente, $61 \%$ e $62 \%$ da força dos homens na extensão e flexão do joelho. Já anteriormente, Murray et al. (16) descreveram valores médios da força dos extensores do joelho de mulheres entre os 20 e os 86 anos de cerca de $74 \%$ daquela observada nos homens de idade semelhante. Também Borges (2) observou que mulheres de dife- 
rentes idades registavam, aproximadamente, $65.7 \%$ e $53 \%$ do torque isocinético para, respectivamente, os extensores e os flexores do joelho comparativamente aos homens do mesmo escalão etário. No entanto, apesar dos vários estudos se referirem aos mais baixos níveis de força encontrados nas mulheres idosas comparativamente aos homens idosos $(2,6,16,22)$, nenhum outro trabalho, segundo o nosso conhecimento, refere que a adaptação da performance muscular ao treino se faz de forma diferenciada entre homens e mulheres $(9,12,22)$. Este facto foi também confirmado no presente estudo, onde, à excepção da flexão do joelho a $180 \%$ seg., não foram observadas diferenças estatisticamente significativas na percentagem de alteração dos valores do momento máximo de força dos membros inferiores após treino entre homens e mulheres. Assim, podemos constatar que, nem as mulheres, nem os homens apresentaram alterações significativas nos níveis de força isocinética dos membros inferiores após 6 meses de treino generalizado. Este "não-efeito" foi provavelmente justificado na insuficiente intensidade e especificidade do protocolo de treino e nos elevados níveis iniciais da amostra. Ou seja, nestas sessões utilizaram-se movimentos necessários para as actividades diárias, mais do que exercícios intensos e específicos de força. Por outro lado, os participantes deste estudo eram indivíduos independentes na sua vida quotidiana, possuindo, provavelmente, um nível de actividade e de força muscular relativamente elevado para a média do seu escalão etário.

\section{CONCLUSÃo}

Os idosos do sexo masculino foram significativamente mais fortes comparativamente ao sexo feminino. Todavia, a percentagem de alteração da força muscular isocinética com o treino não foi significativamente diferente entre os dois sexos.

Assim, este trabalho sugere que, independentemente do sexo, para idosos saudáveis e independentes no seu quotidiano, um programa de actividade física baseado em sessões bi-semanais de "Ginástica de Manutenção" não é suficientemente intenso e/ou específico para induzir alterações que promovam um acréscimo sobre a força da musculatura dos membros inferiores. Sugere-se, por isso, que este tipo de treino generalizado que apresenta inúmeras vantagens, nomeadamente o facto de ser mais motivador e de, geralmente, reflectir melhor as actividades diárias do idoso, dado que trabalha as diferentes componentes da aptidão física, deverá ser acompanhado por treino específico de força, dado esta ser uma capacidade fundamental para a funcionalidade e autonomia do idoso e, consequentemente, para a sua melhor qualidade de vida. Futuros estudos devem considerar a combinação de ambos os tipos de treino.

\section{CORRESPONDÊNCIA \\ Joana Carvalho}

Faculdade de Ciências do Desporto

e de Educação Física

Universidade do Porto

Rua Dr. Plácido Costa, 91

4200.450 Porto

Portugal

jcarvalho@fcdef.up.pt 


\section{REFERÊNCIAS BIBLIOGRÁFICAS}

1. Baecke JAH, Burema J, Frijters JER (1982). A short questionnaire for the measurement of habitual physical activity in epidemiological studies. Am J Clin Nutr 36: 936-942

2. Borges O (1989). Isometric and isokinetic knee extension and flexion torque in men and women aged 20-70. Scand J Rehabil Med 21: 45-53

3. Brill PA, Macera CA, Davis DR, Blair SN, Gordon N (2000). Muscular strength and physical function. Med Sci Sports Exerc 32: 412-416

4. Carter ND, Kannus P, Khan KM (2001). Exercise in prevention of falls in older people. A systematic literature review examining the rationale and evidence. Sports Med 31: 427-438

5. Fiatarone MA, O'Neill EF, Ryan ND, Clements KM, Solares GR, Nelson ME, Roberts SB, Kehayias JJ, Lipsitz LA, Evans WJ (1994). Exercise training and supplementation for physical frailty in very elderly people. $N$ Engl J Med 330: $1769-1775$

6. Frontera WR, Hughes VA, Lutz KJ, Evans WJ (1991). A cross-sectional study of muscle strength and mass in 45 to 78-yr-old men and women. J Appl Physiol 71: 644-650

7. Frontera WR, Meredith CN, O’Reilly KP, Evans WJ (1990) Strength training and determinants of $\mathrm{VO}_{2}$ max in older men. J Appl Physiol 68: 329-333

8. Grimby G, Aniansson A, Hedberg M, Henning G-B, Grangard U, Kvist H (1992). Training can improve muscle strength and endurance in 78- to 84-yr-old men. J Appl Physiol 73: 2517-2523

9. Hakkinen K, Hakkinen A (1995). Neuromuscular adaptations during intensive strength training in middle-aged and elderly males and females. Electromyogr Clin Neurophysiol 35: 137-147

10. Hughes VA, Frontera WR, Wood M, Evans WJ, Dallal GE, Roubenoff R, Fiatarone Singh MA (2001). Longitudinal muscle strength changes in older adults: influence of muscle mass, physical activity and health. J Gerontol 56A: B206-B217

11. Larsson L (1982). Physical training effects on muscle morphology in sedentary males at different ages. Med Sci Sports Exerc 14: 203-206

12. Lemmer JT, Hurlbut DE, Martel GF, Tracy BL, Ivey FM, Metter EJ, Fozard JL, Fleg JL, Hurley BF (2000). Age and gender responses to strength training and detraining. Med Sci Sports Exerc 32: 1505-1512

13. Lindle RS, Metter EJ, Lynch NA, Fleg JL, Fozard JL, Tobin J, Roy TA, Hurley BF (1997). Age and gender comparisons of muscle strength in 654 women and men aged 20-93 yr. J Appl Physiol 83: 1581-1587

14. Lord SR, Ward JA, Williams P, Strudwick M (1995). The effects of a 12-month exercise trial on balance, strength, and falls in older women: a randomized controlled trial. $J$ Am Geriatr Soc 43: 1198-1206

15. Lord SR, Castell S (1994). Physical activity program for older persons: effect on balance, strength, neuromuscular control, and reaction time. Arch Physiol Med Rehabil 75: 648-652

16. Murray MP, Duthie Jr EH, Gambert SR, Sepic SB, Mollinger LA (1985). Age-related differences in knee muscle strength in normal women. $J$ Gerontol 40: 275-280

17. Pollock ML, Wilmore JH (1990). Exercise in Health and Disease: Evaluation and Prescription for Prevention and Rehabilitation. Sauders W B (Ed.), $2^{\text {nd }}$ Ed. Philadelphia, 91160
18. Puggaard L, Pedersen HP, Sandager E, Klitgaard H (1994). Physical conditioning in elderly people. Scand J Med Sci Sports 4: 47-56

19. Salem GJ, Wang M-Y, Young JT, Marion M, Greendale GA (2000). Knee strength and lower- and higher-intensity functional performance in older adults. Med Sci Sports Exerc 32: $1679-1684$

20. Schultz AB (1995). Muscle function and mobility biomechanics in the elderly: an overview of some recent research. J Gerontol 50A: 60-63

21. Skelton DA, Young A, Greig CA, Malbut KE (1995). Effects of resistance training on strength, power, and selected functional abilities of women aged 75 and older. $J$ Am Geriatr Soc 43: 1081-1087

22. Tracy BL, Ivey FM, Hurlbut D, Martel GF, Lemmer JT, Siegel EL, Metter EJ, Fozard JL, Fleg JL, Hurley BF (1999). Muscle quality II. Effects of strength training in 65- to 75yr-old men and women. J Appl Physiol 86: 195-201

23. Vandervoort AA, McComas AJ (1986). Contractile changes in opposing muscles of the human ankle joint with aging. J Appl Physiol 61: 361-367

24. Voorrips LE, Ravelli ACJ, Dongelmans PCA, Deurenberg P, van Staveren WA (1991). A physical activity questionnaire for the elderly. Med Sci Sports Exerc 23: 974-979

25. Westhoff MH, Stemmerik L, Boshuizen HC (2000). Effects of a low-intensity strength-training program on kneeextensor strength and functional ability of frail older people. JAPA 8: 325-342

26. Wilk K (1991). Isokinetic Testing - Setup and Positioning. In Biodex System II Manual, Applications/Operations. Biodex Medical System, Inc, New York, USA. 